\title{
Comparison of Selected Parameters of Redox Homeostasis in Patients with Ataxia-Telangiectasia and Nijmegen Breakage Syndrome
}

\author{
Barbara Pietrucha, ${ }^{1}$ Edyta Heropolitanska-Pliszka, ${ }^{1}$ Mateusz Maciejczyk, ${ }^{2}$ Halina Car, \\ Jolanta Sawicka-Powierza, ${ }^{3}$ Radosław Motkowski, ${ }^{4}$ Joanna Karpinska, ${ }^{5}$ Marta Hryniewicka, ${ }^{5}$ \\ Anna Zalewska, ${ }^{6}$ Malgorzata Pac, ${ }^{1}$ Beata Wolska-Kusnierz, ${ }^{1}$ Ewa Bernatowska, ${ }^{1}$ \\ and Bozena Mikoluc ${ }^{4}$ \\ ${ }^{1}$ Clinical Immunology, The Children's Memorial Health Institute, Av. Dzieci Polskich 20, 04-730 Warsaw, Poland \\ ${ }^{2}$ Department of Experimental Pharmacology, Medical University of Bialystok, Szpitalna 37 Str., 15-295 Bialystok, Poland \\ ${ }^{3}$ Department of Family Medicine, Medical University of Bialystok, Bialystok, Poland \\ ${ }^{4}$ Department of Pediatrics Rheumatology, Immunology, and Metabolic Bone Diseases, Medical University of Bialystok, \\ Waszyngtona 17 Str., 15-274 Bialystok, Poland \\ ${ }^{5}$ Institute of Chemistry, University of Bialystok, Bialystok, Poland \\ ${ }^{6}$ Department of Conservative Dentistry, Medical University of Bialystok, Bialystok, Poland
}

Correspondence should be addressed to Bozena Mikoluc; bozenam@mp.pl

Received 25 August 2017; Revised 21 November 2017; Accepted 3 December 2017; Published 31 December 2017

Academic Editor: Mohamed M. Abdel-Daim

Copyright ( 2017 Barbara Pietrucha et al. This is an open access article distributed under the Creative Commons Attribution License, which permits unrestricted use, distribution, and reproduction in any medium, provided the original work is properly cited.

\begin{abstract}
This study compared the antioxidant status and major lipophilic antioxidants in patients with ataxia-telangiectasia (AT) and Nijmegen breakage syndrome (NBS). Total antioxidant status (TAS), total oxidant status (TOS), oxidative stress index (OSI), and concentrations of coenzyme Q10 (CoQ10) and vitamins A and E were estimated in the plasma of 22 patients with AT, 12 children with NBS, and the healthy controls. In AT patients, TAS (median $261.7 \mu \mathrm{mol} / \mathrm{L}$ ) was statistically lower but TOS $(496.8 \mu \mathrm{mol} / \mathrm{L})$ was significantly elevated in comparison with the healthy group $(312.7 \mu \mathrm{mol} / \mathrm{L}$ and $311.2 \mu \mathrm{mol} / \mathrm{L}$, resp.). Tocopherol $(0.8 \mu \mathrm{g} / \mathrm{mL})$ and CoQ10 $(0.1 \mu \mathrm{g} / \mathrm{mL})$ were reduced in AT patients versus control $(1.4 \mu \mathrm{g} / \mathrm{mL}$ and $0.3 \mu \mathrm{g} / \mathrm{mL}$, resp.). NBS patients also displayed statistically lower TAS levels $(290.3 \mu \mathrm{mol} / \mathrm{L})$, while TOS $(404.8 \mu \mathrm{mol} / \mathrm{L})$ was comparable to the controls. We found that in NBS patients retinol concentration $(0.1 \mu \mathrm{g} / \mathrm{mL})$ was highly elevated and CoQ10 $(0.1 \mu \mathrm{g} / \mathrm{mL})$ was significantly lower in comparison with those in the healthy group. Our study confirms disturbances in redox homeostasis in AT and NBS patients and indicates a need for diagnosing oxidative stress in those cases as a potential disease biomarker. Decreased CoQ10 concentration found in NBS and AT indicates a need for possible supplementation.
\end{abstract}

\section{Introduction}

Ataxia-telangiectasia (AT; OMIM \#208900) and Nijmegen breakage syndrome (NBS; OMIM \#251260) belong to a group of genetically determined primary immunodeficiency disorders (PID) whose course involves defects in DNA repair processes [1]. AT is caused by null mutations in the ATM (ataxia-telangiectasia mutated) gene located on chromosome 11q.26 which encodes a protein of the same name. The main role of ATM protein is the coordination of cellular response to DNA double strand breaks, oxidative stress, signal transduction, and cell-cycle control. The clinical picture of the disease is characterised by cerebellar degeneration, telangiectasia, immunodeficiency, cancer susceptibility, and radiation sensitivity. Experimental studies have provided direct evidence for the presence of mitochondrial dysfunctions in AT 
cells. They have demonstrated that the structural organisation of mitochondria in AT cells is abnormal compared to wild-type cells $[2,3]$.

NBS, similarly to AT, belongs to a group of the XCIND syndrome. The XCIND syndrome is named after Eaton et al. who found distinct hypersensitivity to ionizing (X-ray) irradiation, cancer susceptibility, immunodeficiency, neurological abnormality, and double-strand DNA breakage in these cases [4]. Mutations causative of NBS occur in the NBS1 gene, located on human chromosome 8q21. NBS1 encodes for nibrin, the key regulatory protein of the $\mathrm{R} / \mathrm{M} / \mathrm{N}$ (RAD50/MRE11/NBS1) protein complex which senses and mediates cellular response to DNA damage caused by ionizing radiation $[5,6]$. The chromosome instability in AT and NBS patients results from a defective response to DNA double-strand breaks. In addition, AT and NBS patients display a few common characteristics such as growth retardation, premature aging, and neurodegeneration. It is believed that these symptoms can be caused not only by chromosomal instability and aberrant DNA damage response but also by oxidative stress and mitochondrial abnormalities $[1,2]$.

Recent studies indicate new, alternative sources of ROS and oxidative stress in AT and NBS cells, including NADPH oxidase 4, oxidised low-density lipoprotein (ox-LDL), or poly (ADP-ribose) polymerases (PARP-1, PARP-2, and PARP-3) $[1,7-10]$. Mitochondrial dysfunction such as aberrant structural organisation of mitochondria, excess mitochondrial ROS (mROS) production and mitochondrial injury have also been reported in AT and NBS cells $[1,3,11]$. Valentin-Vega et al. [11] showed increased mROS production in AT cells, which resulted from a decline in the activity of complex I of the electron transport chain in mitochondria. Noteworthy is a study conducted by Weyemi et al. [9] who stressed that NADPH oxidase 4 (the main source of free radicals in the cell) was highly upregulated in AT cells and correlated with higher oxidative damage and apoptosis. To date, however, little is still known about the oxidant/antioxidant abnormalities in AT and NBS patients. Therefore, the purpose of our study was to measure selected parameters of redox homeostasis in patients with AT and NBS. Both disorders are characterised by symptoms typical of the XCIND syndrome, and therefore we intended to evaluate total antioxidant status (TAS), total oxidant status (TOS), and oxidative stress index (OSI) as well as concentrations of the most common lipophilic antioxidants, CoQ10, and vitamins A and E. It appears that the thorough understanding of disturbances in the body's antioxidant defense mechanisms could lead to new therapeutic strategies in AT and NBS, similarly as in other oxidative stress-related genetic disorders.

\section{Materials and Methods}

2.1. Patients. The study included 12 Caucasian children with NBS ( 5 females, 7 males) whose average age was 12 years and 1 month with a confirmed mutation in the NBS1 gene and 22 patients with AT (9 females, 13 males) whose average age was 13 years and 7 months with a confirmed mutation in ATM gene. All study participants were under the medical care of the Department of Immunology at the Children's
Memorial Health Institute in Warsaw, Poland. The patients were included in the study on the basis of their medical history and physical examination, and they were found to be in good health at the time of enrollment (normal biochemical and morphological blood parameters, negative markers of inflammation). Diagnosis was based on clinical symptoms and genetic and biochemical tests according to ESID (European Society for Immunodeficiencies) criteria. None of the patients enrolled in the study were diagnosed with cancer, diabetes, hypertension, and HIV infection. The control group consisted of, respectively, 12 and 22 healthy individuals matched for age and sex. Plasma samples of the study and the control group were collected between February 2016 and January 2017.

The study was approved by the Bioethical Committee at the Medical University, Bialystok, Poland. Parents of all respondents gave informed written consent for their children's participation in the study.

2.2. Determination of Plasma Total Antioxidant Status (TAS), Total Oxidant Status (TOS), and Oxidative Stress Index (OSI). Total antioxidant status (TAS) and total oxidant status (TOS) were determined using commercial colorimetric kits (ImAnOx (TAS/TAC) Kit, Immundiagnostik, Bensheim, Germany and PerOx (TOS/TOC) Kit, Immundiagnostik, Bensheim, Germany, resp.) in accordance with the manufacturer's instructions. The determination of TAS was based on a reaction between antioxidants contained in the sample with exogenous hydrogen peroxide, while the determination of TOS was performed by the reaction of peroxidase with total lipid peroxides in the sample. The resulting coloured products were measured colorimetrically at a wavelength of $450 \mathrm{~nm}$ using Mindray MR-96 Microplate Reader, China. All assays were performed in duplicate samples. To assess the redox balance disorders, we have also used the oxidative stress index (OSI), which may be considered as a gold indicator of oxidative stress in biological systems. OSI was calculated according to the formula OSI $=$ TOS $/$ TAS $\times 100$ [12].

\subsection{Determination of Plasma Vitamin A (All-Trans-Retinol),} Vitamin E (L-Tocopherol), and Coenzyme Q10. Analyses were performed using high performance liquid chromatograph coupled with MS detector equipped with triple quadrupole (Shimadzu LCMS/MS-8040). Ionization was conducted using APCI (atmospheric pressure chemical ionization) mode. Data acquisition and processing were performed using Shimadzu LabSolutions LCMS Software.

The compounds were separated with a Kinetex XB-C18 $100 \mathrm{~A}$ analytical column $(50 \mathrm{~mm} \times 3.0 \mathrm{~mm}, 1.7 \mu \mathrm{m})$. The mobile phase consisted of an isocratic solvent A (methanol) 0.01-2 min and then isocratic solvent B (methanol-n-hexan, $72: 28, v / v) 2.5-6 \mathrm{~min}$. The flow rate was $0.4 \mathrm{~mL} / \mathrm{min}$, and the temperature of the analytical column was $400^{\circ} \mathrm{C}$. The injection volumes of standard and sample solutions were $10 \mu \mathrm{L}$. Acquisition settings and method were optimized by the infusion of a $10 \mu \mathrm{g} / \mathrm{mL}$ solution of each fixed compound. The mass spectrometer was operated in the positive ion atmospheric pressure chemical ionization mode. The APCI temperature was set at $350^{\circ} \mathrm{C}$ and the ion current $4.5 \mu \mathrm{A}$. 
The flow of the drying gas (N2) and the flow of the nebulizing gas were $10 \mathrm{~L} / \mathrm{min}$ and $3 \mathrm{~L} / \mathrm{min}$, respectively. The desolvation line (DL) and heat block temperature was $230^{\circ} \mathrm{C}$.

All analytes were detected in the MS/MS multiple reaction monitoring (MRM) with unit resolution at both Q1 and Q3. The MS conditions for generation of the positive ions are presented in Table 1. The described above chromatographic conditions were used for quantification of target analytes in plasma samples. The chromatogram of real sample is visualized in Figure 1. Plasma samples were prepared according to the procedure published previously [13].

2.4. Statistical Analysis. The examined variable distribution was assessed by means of the Kolmogoroff-Smirnow test. Due to the fact that the tested variables were inconsistent with normal distribution, the Mann-Whitney $U$ test was used. Results are expressed as median, minimum, and maximum. The Spearman's method was applied in assessing correlations between variables. In calculations, the relevance level of $p<0.05$ was accepted as statistically significant, authorising the rejection of individual zero hypotheses. The data were processed using the Polish version of Statistica 12.0 statistical software for PC with Windows.

\section{Results}

Our study demonstrated that in patients with AT plasma TAS levels were statistically lower $(p=0.002)$ and plasma TOS was statistically significantly elevated $(p=0.001)$ in comparison with the control group. Similarly to TOS, OSI was elevated in AT patients $(p=0.001)$ (Figure 2).

Tocopherol plasma concentrations were significantly reduced in AT patients as compared to the control group $(p=0.021)$. The concentrations of endogenous free radical scavenger coenzyme Q10 were statistically decreased in the plasma of AT patients versus the healthy controls $(p=0.001)$. There were no significant differences in the concentrations of plasma retinol between both groups $(p=0.076)$ (Figure 2).

Similarly to the AT group, NBS patients displayed statistically lower plasma TAS levels in comparison with the control group $(p=0.044)$. In contrast to AT, plasma TOS in NBS was not significantly different in comparison with the healthy controls $(p=0.102)$. The OSI value, similarly to AT, was higher in comparison with the controls $(p=0.004)$ (Figure 3).

We demonstrated that in patients with NBS the concentration of vitamin A precursor, beta carotene, was significantly elevated in comparison with that in the control group $(p=0.011)$. The difference in CoQ10 concentration in NBS patients and healthy controls points to a significantly lower level in NBS patients $(p=0.001)$. The concentrations of plasma vitamin $\mathrm{E}$ were similar in the control group and NBS patients $(p=0.582)$ (Figure 3$)$.

It is worth noting that we did not observe statistically significant differences between the evaluated parameters in patients with AT and those with NBS (Table 2). In addition, we did not find significant correlations in the assessed
TABLE 1: The MS conditions for generation of positive ions of the analytes.

\begin{tabular}{lccc}
\hline Compound & $\begin{array}{c}\text { Precursor ion } \\
(\mathrm{m} / \mathrm{z})\end{array}$ & $\begin{array}{c}\text { Product ions } \\
(\mathrm{m} / \mathrm{z})\end{array}$ & $\begin{array}{c}\text { Collision energy } \\
{[\mathrm{eV}]}\end{array}$ \\
\hline \multirow{2}{*}{ Retinol } & 269.10 & 213.20 & -12 \\
& & 93.10 & -23 \\
\hline \multirow{2}{*}{$\alpha$-Tocopherol } & \multirow{2}{*}{429.30} & 165.10 & -25 \\
& & 137.05 & -48 \\
\hline \multirow{2}{*}{ Coenzyme Q10 } & \multirow{2}{*}{863.60} & 197.15 & -45 \\
& & 109.10 & -47 \\
\hline
\end{tabular}

$\mathrm{m} / \mathrm{z}$ : mass-to-charge ratio.

markers of oxidative stress between the study groups and controls.

\section{Discussion}

This study compared the redox balance as well as major lipophilic antioxidants in patients with AT and NBS diseases. We have shown that AT and NBS are associated with impaired redox homeostasis including disturbances in lipophilic free radical scavengers such as coenzyme Q10 and alpha-tocopherol (Figure 4).

We aimed to investigate the oxidant/antioxidant status via the measurement of plasma TAS and TOS, which could provide a systemic reflection of redox homeostasis in biological systems. Concentration of TAS was significantly reduced in AT and NBS patients, which suggests the exhaustion or inefficiency of protective antioxidant systems resulting from the overproduction or enhanced activity of reactive oxygen species (ROS). It is believed that oxidative stress plays a key role in the development of many systemic complications including growth retardation, endocrine abnormalities, neurodegeneration, and premature aging as well as immunodeficiency [14-16], which are the major phenotypic hallmarks in AT and NBS diseases [1]. Our study did not directly demonstrate oxidative stress in patients with AT and NBS, since it would have been necessary to measure oxidative modification products. Nevertheless, we showed the existence of cellular redox abnormalities in the AT $(\downarrow$ TAS, $\uparrow$ OSI, and $\uparrow$ TOS) and NBS groups ( $\downarrow$ TAS and $\uparrow$ OSI), which can constitute the basis for the development of oxidative stress leading to DNA mutations and protein oxidation as well as lipid peroxidation. We would like to emphasise that we did not find significant differences between the researched parameters of redox balance when we compared AT with NBS. A lack of correlation may indicate a similarity of redox imbalance in AT and NBS disorders, although these changes tend to be more severe in patients with AT. However, we recorded significantly elevated TOS levels only in patients with AT and therefore, TOS may be subtle distinguishing between AT and NBS diseases.

There is a consensus in the literature regarding enhanced oxidative stress in neurodegenerative disorders such as Alzheimer's or Parkinson's disease (PD) [17, 18]. Clinical studies have confirmed increased oxidant status and comparable, decreased antioxidant status in patients with $\mathrm{PD}$ [19-21]. It is probable that similar changes occur in the 


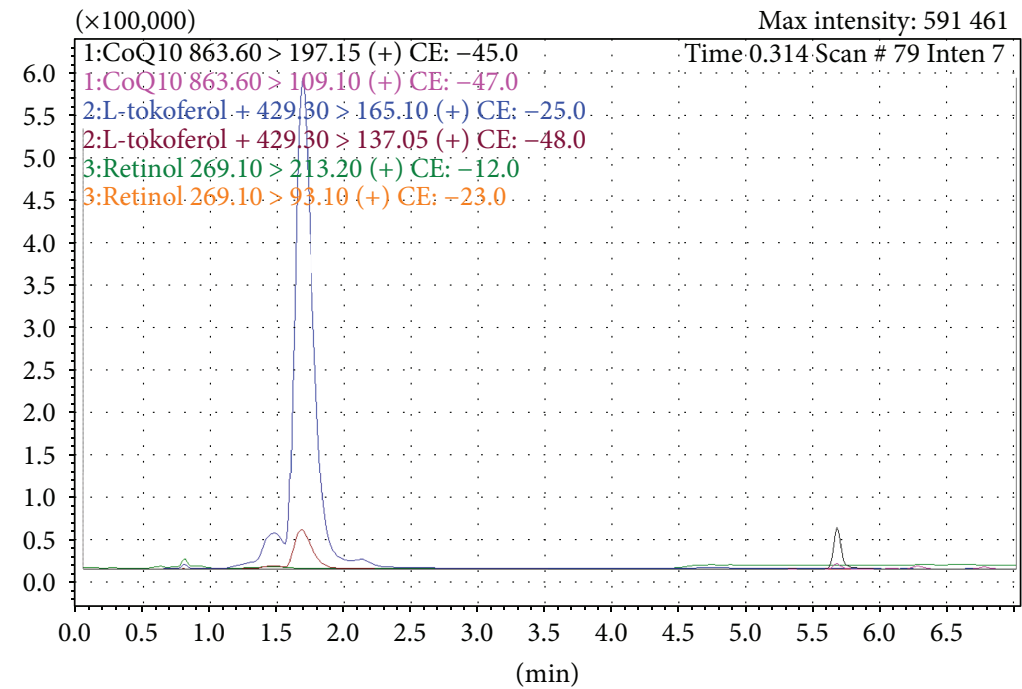

FIGURE 1: Chromatogram of a real sample (plasma).

TAS

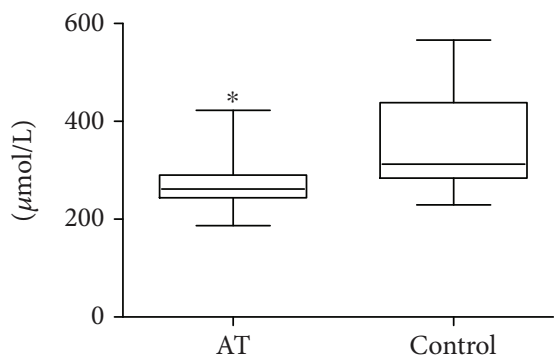

(a)

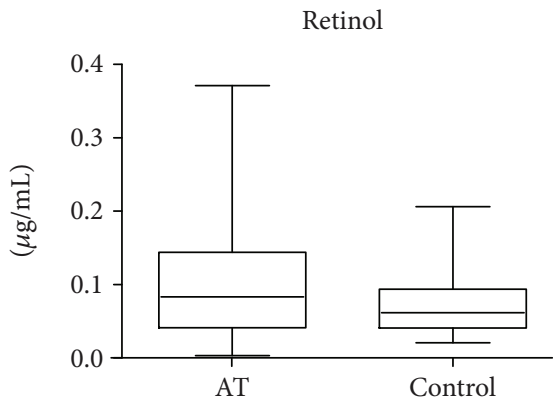

(d)

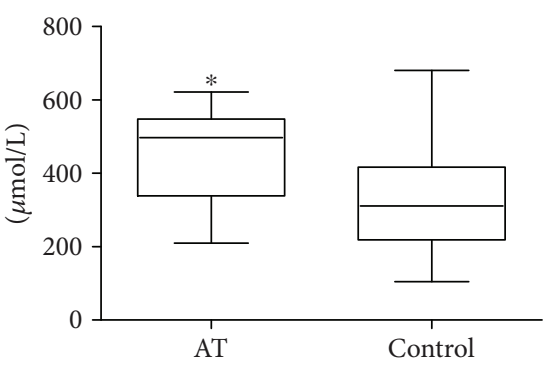

(b)

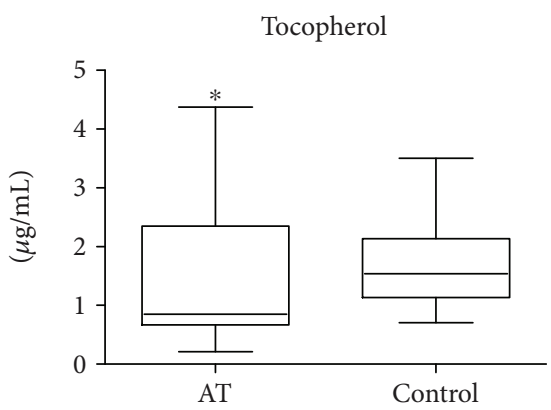

(e)

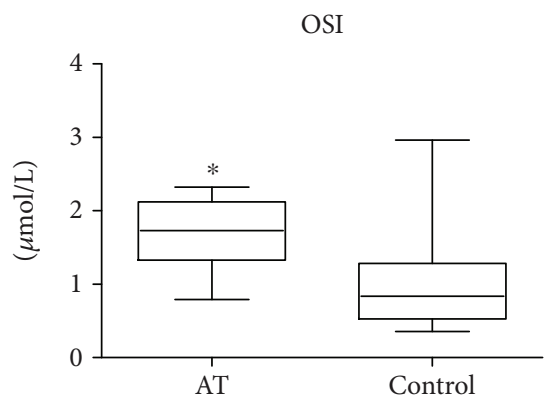

(c)

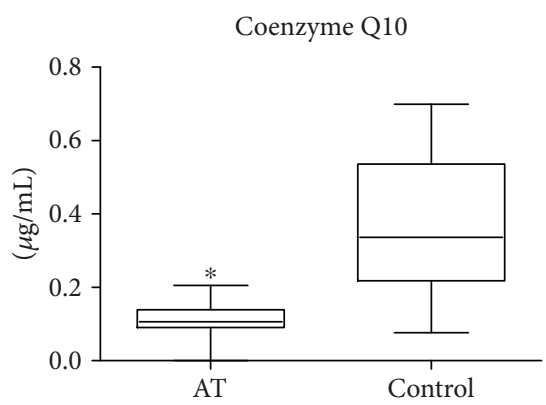

(f)

FIGURE 2: Lipophilic antioxidants and total antioxidant/oxidant status in patients with AT and control group. TAS: total antioxidant status; TOS: total oxidant status; OSI: oxidative stress index; ${ }^{*} p<0.05$.

course of AT and NBS. However, the available literature contains limited data regarding oxidative stress/redox disturbances in those cases [1]. Additionally, it should be noted that the measurement of plasma TAS and TOS provides information about antioxidant properties conditioned primarily by low molecular weight (LWMA) hydrophilic antioxidants (e.g., uric acid (UA) and ascorbic acid (AA) as well as thiol groups), but not lipophilic antioxidants [22]. Taking this into consideration, we also decided to measure levels of major lipophilic free radical scavengers (CoQ10, vitamins $\mathrm{A}$ and $\mathrm{E})$.
Our study confirm decreased CoQ10 concentration both in AT and NBS. Coenzyme Q10 (also called ubiquinone) is a lipid soluble benzoquinone which is a key component of the mitochondrial respiratory chain for adenosine triphosphate (ATP) synthesis [23]. It has been demonstrated that most of the CoQ10 in the human body is produced endogenously; $25 \%$ of the stores are obtained from dietary intake [24]. Coenzyme Q10 deficiency results not only in abnormal respiratory chain function with inadequate cellular energy production, increased generation of free radicals, and degradation of mitochondria but also impacts on the immune 
TAS

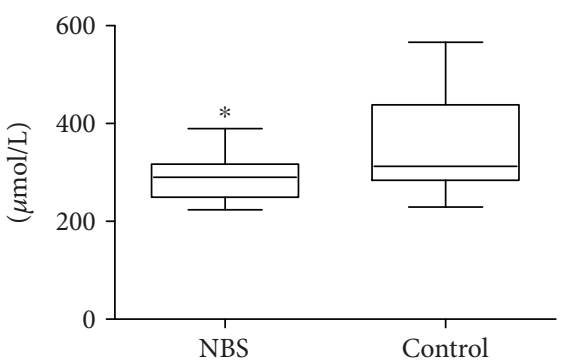

(a)

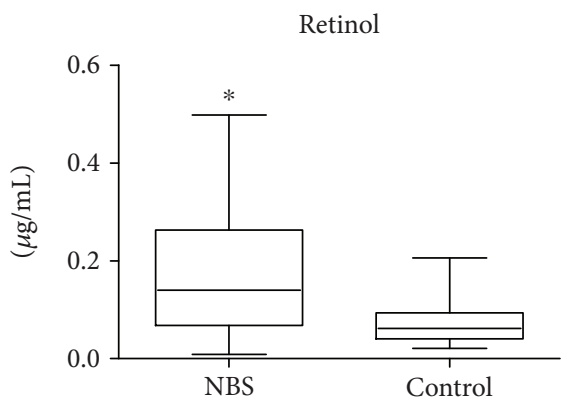

(d)
TOS

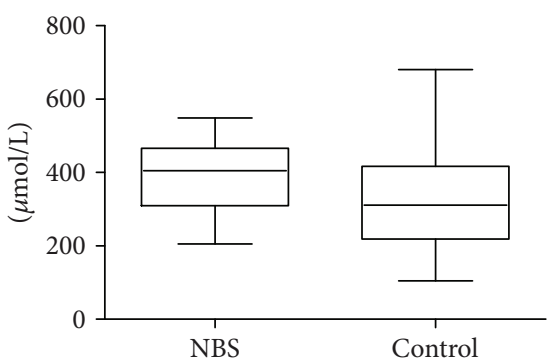

(b)

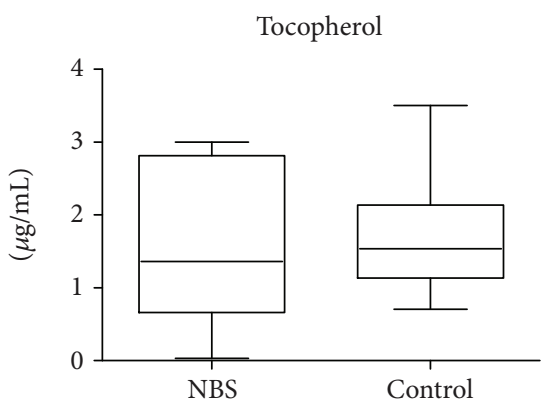

(e)
OSI

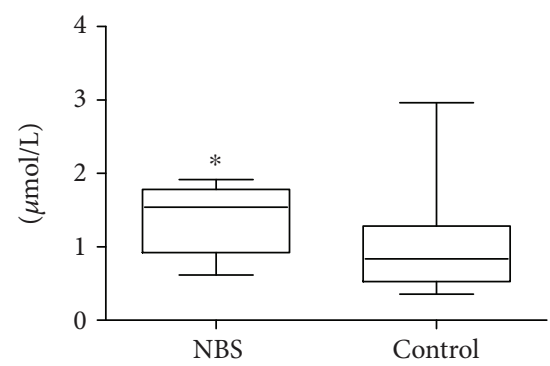

(c)

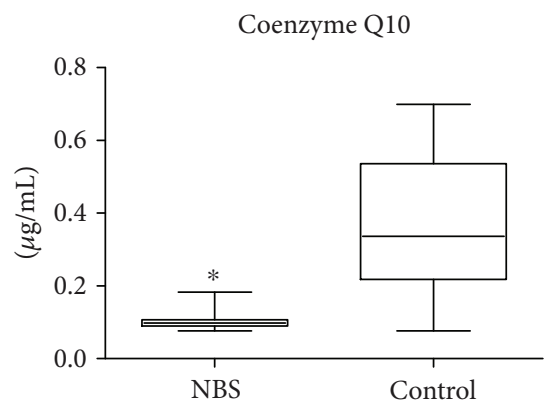

(f)

FIGURE 3: Lipophilic antioxidants and total antioxidant/oxidant status in patients with NBS and control group. TAS: total antioxidant status; TOS: total oxidant status; OSI: oxidative stress index; ${ }^{*} p<0.05$.

TABle 2: Differences between lipophilic antioxidants and total antioxidant/oxidant status in patients with AT and NBS.

\begin{tabular}{lcccr}
\hline \multirow{2}{*}{ Parameters examined } & \multicolumn{2}{c}{ AT group } & \multicolumn{2}{c}{ NBS group } \\
& Median & Min-max & 0.140 & $0.009-0.498$ \\
\hline Retinol $(\mu \mathrm{g} / \mathrm{mL})$ & 0.083 & $0.003-0.371$ & 1.361 & $0.032-3.003$ \\
$\alpha$-Tocopherol $(\mu \mathrm{g} / \mathrm{mL})$ & 0.847 & $0.213-4.371$ & 0.098 & $0.077-0.183$ \\
Coenzyme Q10 $(\mu \mathrm{g} / \mathrm{mL})$ & 0.108 & $0.085-0.206$ & 290.288 & $223.966-389.895$ \\
TAS $(\mu \mathrm{mol} / \mathrm{L})$ & 261.745 & $186.852-422.515$ & 404.795 & $204.741-548.632$ \\
TOS $(\mu \mathrm{mol} / \mathrm{L})$ & 496.839 & $209.803-621.354$ & 1.543 & 0.290 \\
OSI & 1.730 & $0.790-2.320$ & $0.615-1.915$ & 0.094 \\
\hline
\end{tabular}

TAS: total antioxidant status; TOS: total oxidant status; OSI: oxidative stress index.

system [25]. Literature reports demonstrate that CoQ10 supplementation has improved CD4 T cell counts in patients with AIDS and outcomes in herpes and HPV infections. Furthermore, leukocyte activity is conditioned, inter alia, by proper CoQ10 activity [25]. CoQ10 deficiency may contribute to the abnormal function of mitochondria in the course of diseases such as AIDS, Alzheimer's disease, Parkinson's disease, and cancer [26, 27]. Similar disturbances have been observed in AT and NBS cells, which may suggest a potential involvement of CoQ10 in the pathogenesis of these disorders [1]. Considering the protective action of CoQ10 in neurodegenerative processes and its role in the immune system, decreased CoQ10 concentration in AT and NBS patients indicates a need for monitoring its concentration and potential supplementation. At this stage, we are unable to answer the question to what degree clinical symptoms observed in AT, such as cerebellar degeneration, immunodeficiency, cancer susceptibility, and radiation sensitivity, result from oxidative stress disturbances observed in our study. Are these disturbances primary or secondary? However, they undeniably confirm CoQ10 involvement in the pathomechanism of the observed changes. It should be also emphasised that rare autosomal recessive disorder, CoQ10 deficiency (mutation in CABC1; COQ2; COQ9; PDSS1; PDSS2 genes), is frequently associated with seizures, cognitive decline, pyramidal track signs, myopathy, and prominent cerebellar ataxia $[28,29]$. Some of these symptoms occur in both AT and NBS. Additionally, it is very likely that, in AT and NBS patients, a decrease in CoQ10 levels may be associated with impaired DNA repair mechanisms, similarly as in other bioenergetics disorders such as xeroderma pigmentosum (XP), Cockayne Syndrome (CS), Fanconi anaemia (FA), and HutchinsonGilford syndrome (HGS) [1].

Deficiency in lipid soluble antioxidants has been demonstrated in various conditions such as eating disorders, nicotine addiction, chronic diseases, and aging. Antioxidant vitamins and trace elements contribute to maintaining an effective immune response [30-33]. Treatments are also 


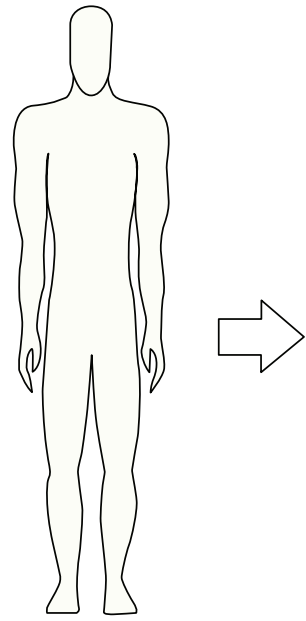

22 AT patients

12 NBS patients

22 healthy controls

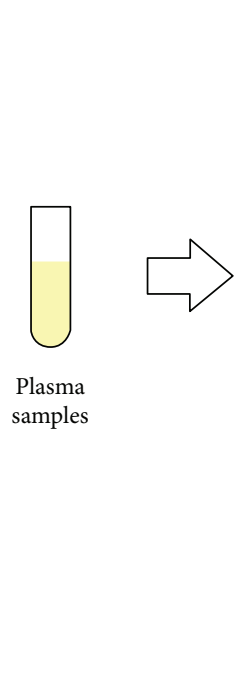

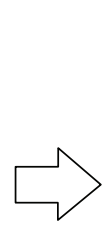

Vitamin A (all-trans-retinol)

Vitamim E (L-tocopherol)

Coenzyme Q10

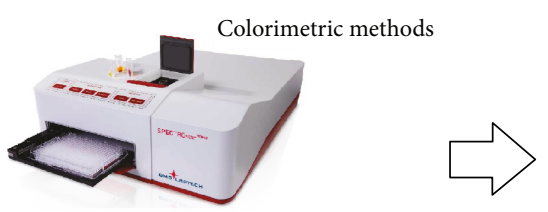

Total antioxidant status (TAS)

Total oxidant status (TOS)

Oxidative stress index (OSI)
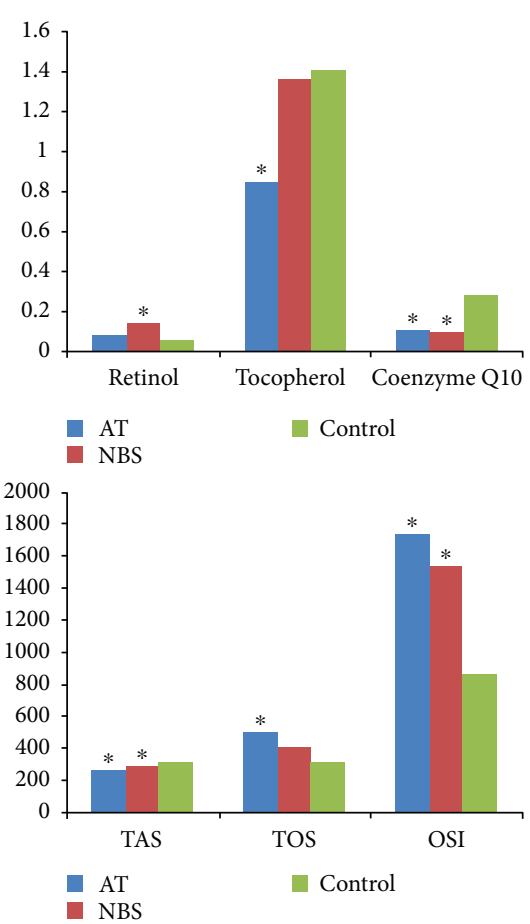

FIGURE 4: Graphical presentation of the study. ${ }^{*} p<0.05$ versus control.

available for a few autosomal recessive ataxias: vitamin $\mathrm{E}$ therapy for ataxia with vitamin E deficiency (AVED), chenodeoxycholic acid for cerebrotendinous xanthomatosis, CoQ10 for CoQ10 deficiency, and dietary restriction of phytanic acid for Refsum disease. Idebenone may ameliorate the cardiac and neurological manifestations of Friedreich ataxia (FRDA). No other specific treatments exist for hereditary ataxias [34]. Unfortunately, no sufficiently effective causal therapy is available for AT and NBS patients at present. Our results indicate a need for introducing CoQ10 and tocopherol into AT and NBS treatment, taking into consideration their plasma levels.

Alpha-tocopherol primarily inhibits the production of new free radicals and thus might help in preventing or delaying chronic diseases associated with reactive oxygen species molecules [35]. Decreased vitamin E concentration in AT may result in a decline in the body's antioxidant activity and anti-inflammatory as well as cell-mediated and humoral immune functions. In AT cases, we found decreased concentrations of both CoQ10 and vitamin E, which enhance disturbances in the redox balance and may increase ROS production, accumulation of damaged mitochondrial DNA (mDNA), and progressive respiratory chain dysfunction. The few experimental studies found in the available literature report that disturbances in redox processes also occur in NBS [1]. Our study of NBS cases demonstrated decreased TAS and enhanced OSI on the one hand and a reduced concentration of CoQ10 and increased concentration of vitamin A, on the other hand. Despite the fact that the interpretation of obtained results is difficult at this stage of research advancement due to a lack of comparable studies, we would like to emphasise that we did not find any differences in the tested parameters between AT and NBS.
To date, only few studies have evaluated the antioxidant defenses in AT and NBS patients $[1,36]$. In one of the first reports on the subject, Reichenbach et al. showed a decrease in the levels of total antioxidant capacity, vitamin A, and vitamin $\mathrm{E}$ in $\mathrm{AT}$ patients. In contrast to our study, CoQ10 levels in AT were not significantly reduced [37]. In our study patients with AT, we found not only significantly reduced levels of CoQ10 but also of $\alpha$-tocopherol. However, retinol concentration, in line with studies by Da Silva et al., was similar to the control group [38]. It is probable that the aforementioned dissimilarities result from differences in the size of study groups and research methodologies. At this point, it should be emphasized that our study is the first in which the largest group of AT and NBS patients took part.

In summary, we demonstrated a significant decrease in the level of plasma TAS in AT and NBS patients as well as an increase in the oxidative stress index. It appears that disturbances in the body's antioxidant defense mechanisms may lead to oxidative damage and altered cellular redox homeostasis and thus affect clinical manifestations of AT and NBS phenotypes. Redox imbalance in patients with AT and NBS appears to exist at a comparable level. However, a significant increase in total oxidant status (TOS) was demonstrated only in patients with AT in whom plasma TOS may be a subtle distinguishing feature of AT and NBS disorders. We are considering the theory that antioxidant supplementation with tocopherol and CoQ10 may be one of the factors enhancing treatment efficacy in AT and NBS diseases and improving patients' clinical status. Further studies regarding CoQ10 in AT and NBS are needed in order to assess its concentration not only in the blood but also in selected tissues and to evaluate the dynamics of change in relation to disease duration. The issue of a possible link between 
CoQ10 deficiency and the occurrence of mutations responsible for CoQ10 deficiency remains open.

When analyzing the results of our research, one must bear in mind its limitations. We evaluated only selected parameters of redox balance in patients with AT and NBS, so determination of other markers may lead to other conclusions and clinical applications. Additionally, blood oxidative stress biomarkers cannot be mechanistically informative for some reasons. Firstly, blood and the respective tissue under study do not share common redox components and/or pathways (e.g., lack of mitochondria in erythrocytes and plasma). Secondly, both plasma and blood cells can autonomously produce significant amounts of $\operatorname{ROS}[15,16]$. However, it should be also emphasized that extremely strong point of our study is the large number of patients in the study groups compared to previously published works. Our research may also provide a new practical aspects for clinicians.

\section{Conclusions}

(1) Our study confirms disturbances in redox homeostasis in AT and NBS patients and indicates a need for diagnosing oxidative stress in those cases as a potential disease biomarker.

(2) The statistically significantly decreased coenzyme Q10 concentration found in NBS and AT indicates a need for possible supplementation aimed at remedying the diagnosed deficiencies in those individuals.

\section{Abbreviations}

$\begin{array}{ll}\text { APCI: } & \begin{array}{l}\text { Atmospheric pressure chemical } \\ \text { ionization }\end{array} \\ \text { AT: } & \text { Ataxia-telangiectasia } \\ \text { ATM: } & \text { Ataxia-telangiectasia mutated } \\ \text { CoQ10: } & \text { Coenzyme Q10 } \\ \text { ESID: } & \text { European Society for } \\ & \text { Immunodeficiencies } \\ \text { LWMA: } & \text { Low molecular weight hydrophilic } \\ & \text { antioxidants } \\ \text { MRM: } & \text { MS/MS multiple reaction monitoring } \\ \text { NBS: } & \text { Nijmegen breakage syndrome } \\ \text { OSI: } & \text { Oxidative stress index } \\ \text { PARP: } & \text { Poly (ADP-ribose) polymerase } \\ \text { PID: } & \text { Primary immunodeficiency disorder } \\ \text { ROS: } & \text { Reactive oxygen species } \\ \text { TAS: } & \text { Total antioxidant status } \\ \text { TOS: } & \text { Total oxidant status } \\ \text { XCIND syndrome: } & \text { Hypersensitivity to ionizing (X-ray) } \\ & \text { irradiation, cancer susceptibility, } \\ & \text { immunodeficiency, neurological } \\ & \text { abnormality, and double-strand } \\ & \text { DNA breakage. }\end{array}$

\section{Conflicts of Interest}

The authors declare that they have no conflicts of interest.

\section{Authors' Contributions}

Barbara Pietrucha, Edyta Heropolitanska-Pliszka, and Mateusz Maciejczyk had equal participation.

\section{Acknowledgments}

The investigation was supported by Grant nos. 143-26591L and N/ST/ZB/16/001/1126 from the Medical University in Bialystok.

\section{References}

[1] M. Maciejczyk, B. Mikoluc, B. Pietrucha et al., "Oxidative stress, mitochondrial abnormalities and antioxidant defense in ataxia-telangiectasia, bloom syndrome and Nijmegen breakage syndrome," Redox Biology, vol. 11, pp. 375383, 2017.

[2] F. V. Pallardó, A. Lloret, M. Lebel et al., "Mitochondrial dysfunction in some oxidative stress-related genetic diseases: ataxia-telangiectasia, down syndrome, Fanconi anaemia and Werner syndrome," Biogerontology, vol. 11, no. 4, pp. 401419, 2010.

[3] M. Ambrose, J. V. Goldstine, and R. A. Gatti, "Intrinsic mitochondrial dysfunction in ATM-deficient lymphoblastoid cells," Human Molecular Genetics, vol. 16, no. 18, pp. 21542164, 2007.

[4] J. S. Eaton, Z. P. Lin, A. C. Sartorelli, N. D. Bonawitz, and G. S. Shadel, "Ataxia-telangiectasia mutated kinase regulates ribonucleotide reductase and mitochondrial homeostasis," The Journal of Clinical Investigation, vol. 117, no. 9, pp. 27232734, 2007.

[5] K. H. Chrzanowska, W. J. Kleijer, M. Krajewska-Walasek et al., "Eleven Polish patients with microcephaly, immunodeficiency, and chromosomal instability: the Nijmegen breakage syndrome," American Journal of Medical Genetics, vol. 57, no. 3, pp. 462-471, 1995.

[6] S. Mizutani and M. Takagi, "XCIND as a genetic disease of X-irradiation hypersensitivity and cancer susceptibility," International Journal of Hematology, vol. 97, no. 1, pp. 3742, 2013.

[7] S. Squadrone, P. Brizio, C. Mancini et al., "Blood metal levels and related antioxidant enzyme activities in patients with ataxia telangiectasia," Neurobiology of Disease, vol. 81, pp. 162-167, 2015.

[8] M. Semlitsch, R. E. Shackelford, S. Zirkl, W. Sattler, and E. Malle, "ATM protects against oxidative stress induced by oxidized low-density lipoprotein," DNA Repair, vol. 10, no. 8, pp. 848-860, 2011

[9] U. Weyemi, C. E. Redon, T. Aziz et al., "NADPH oxidase 4 is a critical mediator in ataxia telangiectasia disease," Proceedings of the National Academy of Sciences of the United States of America, vol. 112, no. 7, pp. 2121-2126, 2015.

[10] H. Krenzlin, I. Demuth, B. Salewsky et al., "DNA damage in Nijmegen breakage syndrome cells leads to PARP hyperactivation and increased oxidative stress," PLoS Genetics, vol. 8, no. 3, article e1002557, 2012.

[11] Y. A. Valentin-Vega, K. H. MacLean, J. Tait-Mulder et al., "Mitochondrial dysfunction in ataxia-telangiectasia," Blood, vol. 119, no. 6, pp. 1490-1500, 2012.

[12] J. Borys, M. Maciejczyk, A. J. Krętowski et al., "The redox balance in erythrocytes, plasma, and periosteum of patients 
with titanium fixation of the jaw," Frontiers in Physiology, vol. 8, 2017.

[13] J. Karpińska, B. Mikołuć, R. Motkowski, and J. PiotrowskaJastrzebska, "HPLC method for simultaneous determination of retinol, $\alpha$-tocopherol and coenzyme Q10 in human plasma," Journal of Pharmaceutical and Biomedical Analysis, vol. 42, no. 2, pp. 232-236, 2006.

[14] O. I. Aruoma, "Free radicals, oxidative stress, and antioxidants in human health and disease," Journal of the American Oil Chemists' Society, vol. 75, no. 2, pp. 199-212, 1998.

[15] M. Valko, D. Leibfritz, J. Moncol, M. T. D. Cronin, M. Mazur, and J. Telser, "Free radicals and antioxidants in normal physiological functions and human disease," The International Journal of Biochemistry \& Cell Biology, vol. 39, no. 1, pp. 44-84, 2007.

[16] B. Uttara, A. V. Singh, P. Zamboni, and R. T. Mahajan, "Oxidative stress and neurodegenerative diseases: a review of upstream and downstream antioxidant therapeutic options," Current Neuropharmacology, vol. 7, no. 1, pp. 65-74, 2009.

[17] A. Arikanoglu, E. Akil, S. Varol et al., "Relationship of cognitive performance with prolidase and oxidative stress in Alzheimer disease," Neurological Sciences, vol. 34, no. 12, pp. 2117-2121, 2013.

[18] A. Kirbas, S. Kirbas, M. C. Cure, and A. Tufekci, "Paraoxonase and arylesterase activity and total oxidative/anti-oxidative status in patients with idiopathic Parkinson's disease," Journal of Clinical Neuroscience, vol. 21, no. 3, pp. 451-455, 2014.

[19] M. Y. Sherman and A. L. Goldberg, "Cellular defenses against unfolded proteins: a cell biologist thinks about neurodegenerative diseases," Neuron, vol. 29, no. 1, pp. 15-32, 2001.

[20] T. B. Shea, Y. L. Zheng, D. Ortiz, and H. C. Pant, "Cyclindependent kinase 5 increases perikaryal neurofilament phosphorylation and inhibits neurofilament axonal transport in response to oxidative stress," Journal of Neuroscience Research, vol. 76, no. 6, pp. 795-800, 2004.

[21] A. K. Verma, J. Raj, V. Sharma, T. B. Singh, S. Srivastava, and R. Srivastava, "Plasma Prolidase activity and oxidative stress in patients with Parkinson's disease," Parkinson's Disease, vol. 2015, Article ID 598028, 6 pages, 2015.

[22] C. Rice-Evans and N. J. Miller, "Total antioxidant status in plasma and body fluids," Methods in Enzymology, vol. 234, pp. 279-293, 1994.

[23] L. Ernster and G. Dallner, "Biochemical, physiological and medical aspects of ubiquinone function," Biochimica et Biophysica Acta (BBA) - Molecular Basis of Disease, vol. 1271, no. 1, pp. 195-204, 1995.

[24] H. N. Bhagavan and R. K. Chopra, "Coenzyme Q10: absorption, tissue uptake, metabolism and pharmacokinetics," Free Radical Research, vol. 40, no. 5, pp. 445-453, 2006.

[25] S. Farough, A. Karaa, M. A. Walker et al., "Coenzyme Q10 and immunity: a case report and new implications for treatment of recurrent infections in metabolic diseases," Clinical Immunology, vol. 155, no. 2, pp. 209-212, 2014.

[26] J. Garrido-Maraver, M. D. Cordero, M. Oropesa-Ávila et al., "Coenzyme Q10 therapy," Molecular Syndromology, vol. 5, no. 3-4, pp. 187-197, 2014.

[27] I. P. Hargreaves, "Coenzyme Q10 as a therapy for mitochondrial disease," The International Journal of Biochemistry \& Cell Biology, vol. 49, pp. 105-111, 2014.
[28] C. Lamperti, M. H. a Naini, D. C. De Vivo et al., "Cerebellar ataxia and coenzyme Q10 deficiency," Neurology, vol. 60, no. 7, pp. 1206-1208, 2003.

[29] R. Montero, M. Pineda, A. Aracil et al., "Clinical, biochemical and molecular aspects of cerebellar ataxia and coenzyme Q10 deficiency," Cerebellum, vol. 6, no. 2, pp. 118-122, 2007.

[30] J. A. Drisko, "The use of antioxidants in transmissible spongiform encephalopathies: a case report," Journal of the American College of Nutrition, vol. 21, no. 1, pp. 22-25, 2002.

[31] D. Q. Pham and R. Plakogiannis, "Vitamin E supplementation in Alzheimer's disease, Parkinson's disease, tardive dyskinesia, and cataract: part 2," The Annals of Pharmacotherapy, vol. 39, no. 12, pp. 2065-2072, 2005.

[32] C. Ramassamy, "Emerging role of polyphenolic compounds in the treatment of neurodegenerative diseases: a review of their intracellular targets," European Journal of Pharmacology, vol. 545 , no. 1, pp. 51-64, 2006.

[33] D. Brambilla, C. Mancuso, M. R. Scuderi et al., "The role of antioxidant supplement in immune system, neoplastic, and neurodegenerative disorders: a point of view for an assessment of the risk/benefit profile," Nutrition Journal, vol. 7, no. 1, p. 29, 2008.

[34] S. Jayadev and T. D. Bird, "Hereditary ataxias: overview," Genetics in Medicine, vol. 15, no. 9, pp. 673-683, 2013.

[35] S. Rizvi, S. T. Raza, F. Ahmed, A. Ahmad, S. Abbas, and F. Mahdi, "The role of vitamin $\mathrm{E}$ in human health and some diseases," Sultan Qaboos University Medical Journal, vol. 14, no. 2, pp. 157-165, 2014.

[36] A. Lloret, R. Calzone, C. Dunster et al., "Different patterns of in vivo pro-oxidant states in a set of cancer- or aging-related genetic diseases," Free Radical Biology \& Medicine, vol. 44, no. 4, pp. 495-503, 2008.

[37] J. Reichenbach, R. Schubert, C. Schwan, K. Müller, H. J. Böhles, and S. Zielen, "Anti-oxidative capacity in patients with ataxia telangiectasia," Clinical and Experimental Immunology, vol. 117, no. 3, pp. 535-539, 1999.

[38] R. da Silva, E. C. dos Santos-Valente, F. Burim Scomparini, R. O. Saccardo Sarni, and B. T. Costa-Carvalho, "The relationship between nutritional status, vitamin a and zinc levels and oxidative stress in patients with ataxia-telangiectasia," Allergologia et Immunopathologia, vol. 42, no. 4, pp. 329-335, 2014. 


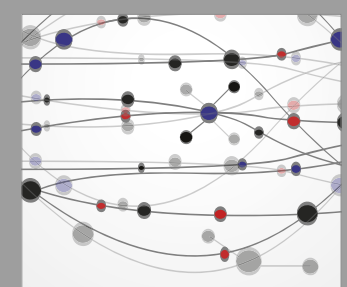

The Scientific World Journal
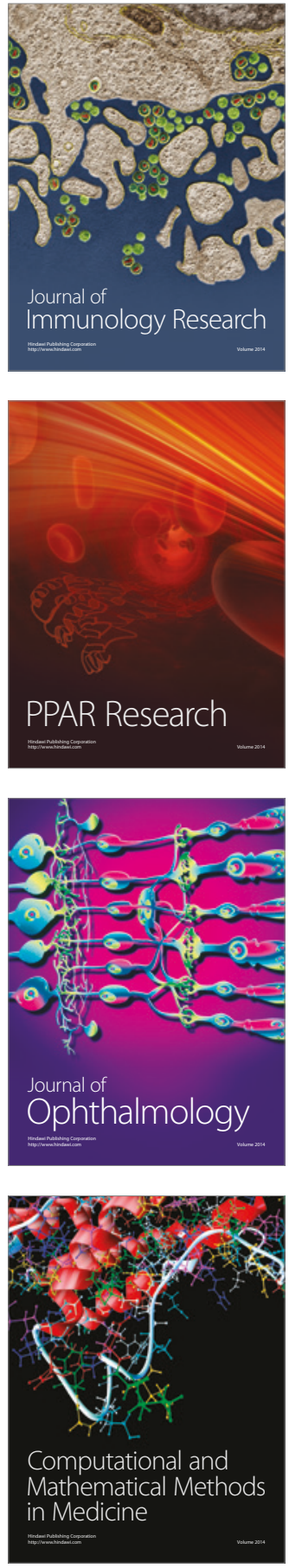

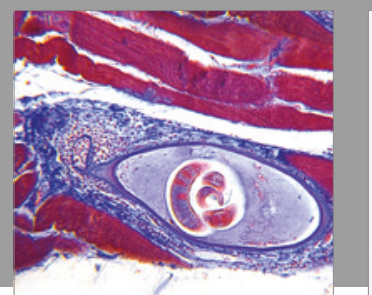

Gastroenterology Research and Practice
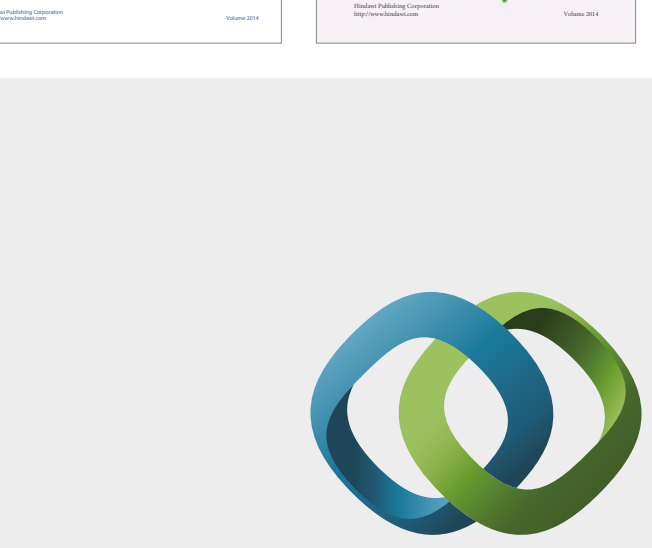

\section{Hindawi}

Submit your manuscripts at

https://www.hindawi.com
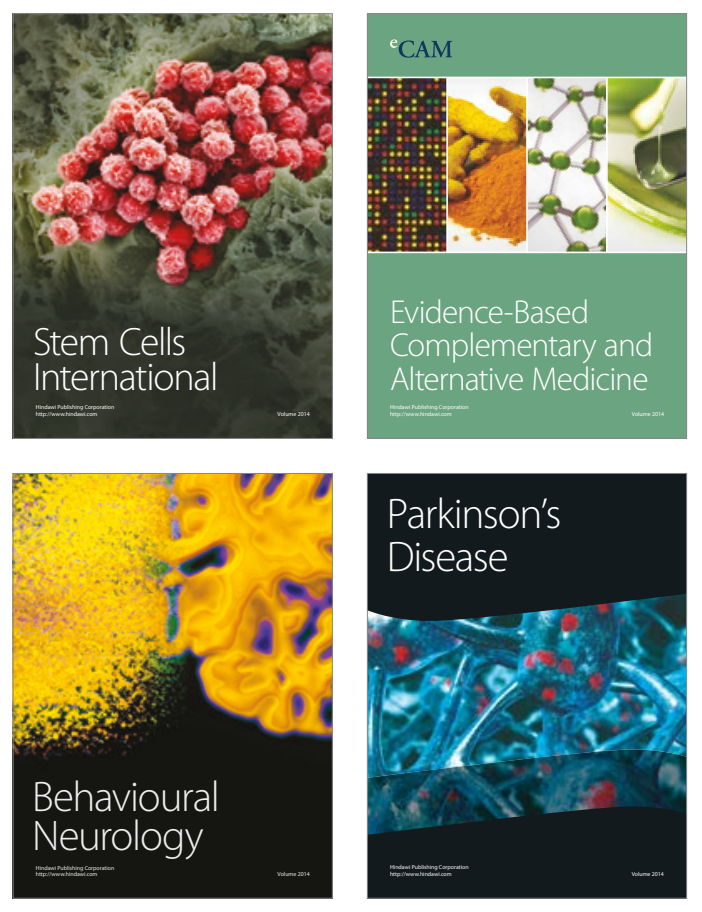
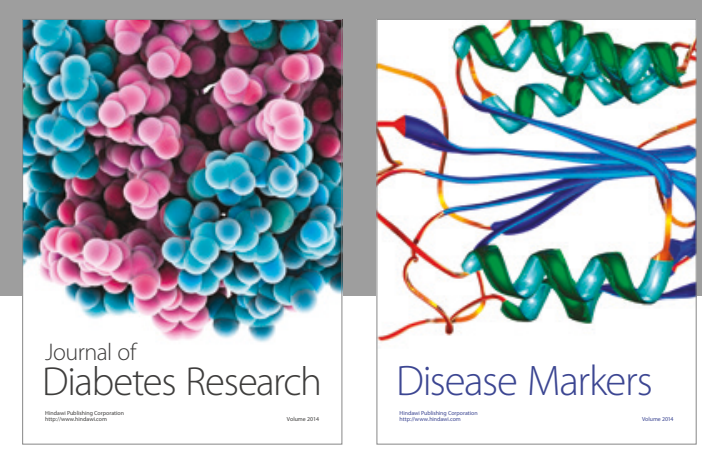

Disease Markers
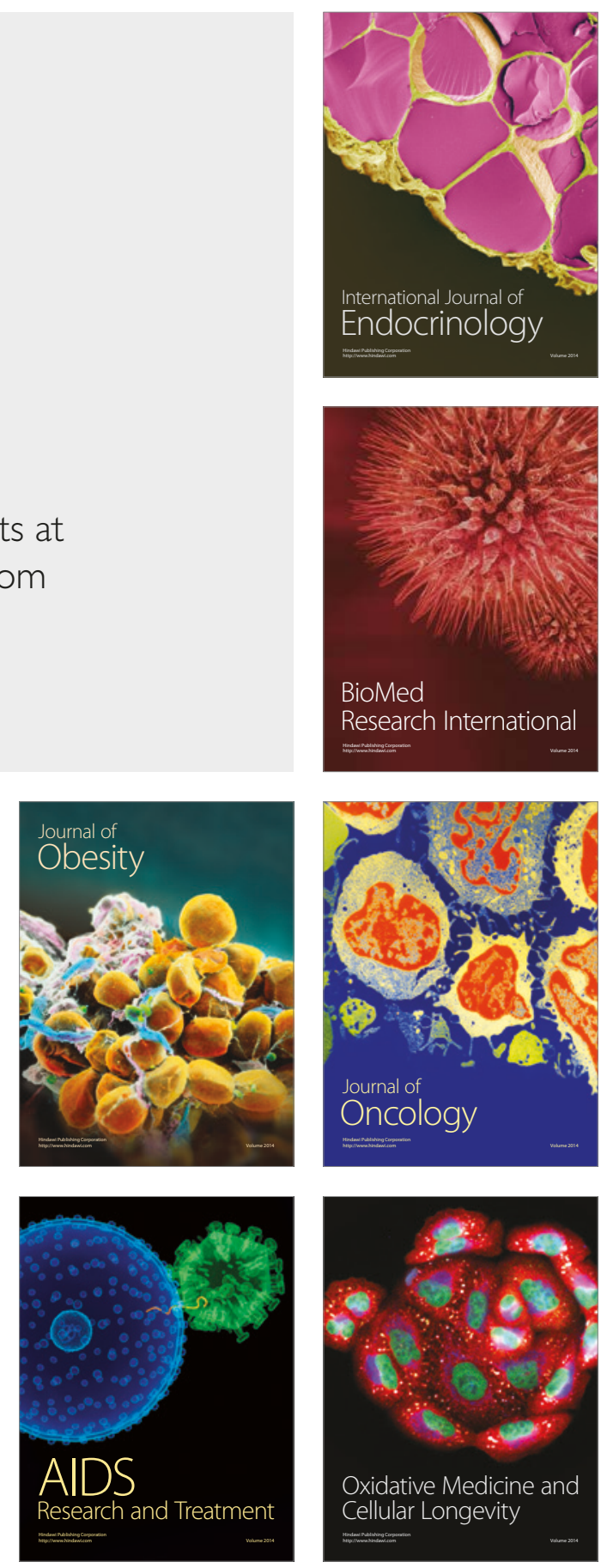